\title{
Gangrene: Types, Characteristics and Treatment
}

\section{Kumar $A^{*}$}

School of Biotechnology, Devi Ahilya University, India

Mini review

Volume 5 Issue 2

Received Date: March 18, 2020

Published Date: April 06, 2020

DOI: $10.23880 /$ cdoaj-16000211

\section{Abstract}

Gangrene is characterized by necrosis of a part of body due to lack of blood and oxygen supplies followed by tissue death. In many gangrene cases, wound in the affected body part get infected with a pathogenic bacteria. Although any part of the body may get affected but feet, legs, fingers and hands are more prone to this disease since these get wounded more frequently, and if care is not taken, wound gets infected by pathogenic bacteria. This disease has been reported both in male and female humans. There are number of factors responsible to stimulate gangrene. Three different types of gangrene viz. dry, wet and gas gangrenes are there. There is also a specific Fournier gangrene under gas gangrene which occurs in genitalia or perineum. In the present mini-review, these different types of gangrene have been briefly discussed. Causes for these different types of gangrene have also been discussed. Gas gangrene is considered to be the most dangerous out of all the three diseases. There is a main pathogenic microbe, Clostridium perfringens which causes wet as well as gas gangrenes. The recent work on this microbe has also been briefly discussed.

Keywords: Gangrene; Dry gangrene; Wet gangrene; Gas gangrene; Fournier gangrene; Clostridium perfringens

\section{Introduction}

When there is necrosis of any part of the body due to lack of blood and oxygen supplies, it is called 'gangrene'. It is a type of tissue death. Although any part of the body may get necrosis, it has been more frequently reported in feet, toe of feet, hands and fingers. These are those parts which are wounded more frequently after getting hurt. Gangrene has been reported both in male and female humans. It is also sometimes reported in the internal parts of the body. In some cases, it is reported in a part inside the abdomen. It has been shown that those persons who don't eat meal properly, diabetic patients and older people in whose filling of wounds take more time, are more prone to the disease than others. The phenotypic symptoms of gangrene when in visible parts are change in color of the skin to black or red, swelling, skin breakdown, numbness etc (https://www.nhs. uk/conditions/gangrene/symptoms/). Many times, patients also suffer from fever or sepsis (https://en.wikipedia.org/ wiki/Gangrene).

\section{Factors Affecting The Disease}

There may be many factors responsible for developing gangrene. It has been speculated that arteriosclerosis obliterans (a pathological condition when arteries carrying blood from heart to other parts of the body become thick and stiff resulting restriction in blood flow) and thromboangiitis obliterans (an inflammatory vasculo-disease, also known as Buerger disease, where inflammation starts within tunica intima and it affects small and medium sized arteries and veins in the body leading to prothrombotic state and thereafter vaso-occlusive phenomena) are the main causes for gangrene. Besides, following pathological diseases may also stimulate formation of gangrene in the body:

- Raynaud's disease (a rare disease of blood vessels in the toes and fingers where narrowing of blood vessels occurs in cold or stressed condition. It results not in flow of blood up to the surface of the skin and affected area turns white and blue) 
- Diabetes (increased level of glucose in blood and urine)

- Polycythemia (a type of blood cancer where increase in RBCs and decrease in plasma volume occurs)

- Sickle Cell Anaemia (a disorder of haemoglobin present in RBCs, changed haemoglobin is called as haemoglobin $\mathrm{S}$; and it distorts RBCs into a sickle or crescent shape. These distorted RBCs are not able to move smoothly through blood vessels and block the blood flow and oxygen supply to different parts of the body)

- Arterial or venous embolism (when blood thrombus (clot) forms in artery or vein resulting hurdle in blood flow)

- Ergotism and Frostbite [poisoning in humans on eating ergot fungus (Claviceps sp.) infected food like rye bread which produces alkaloids; and frostbite is freezing of the skin or other tissues like toes, fingers, hands, feet etc upon exposure to chilled temperature resulting in numbness)

- Trauma (an incident which causes physical, emotional or psychological harm to the body)

- Hypogammaglobulinemia (an immune disorder when reduction in gamma globulins of all types including antibodies occurs)

- Collagen disease (when defects in collagen occur)

- Tuberculosis (infection of Mycobacterium tuberculosis in lungs)

- Erysipelas and surgical infections of palms (a bacterial infection caused by Streptococcus sp. on scratches, most common on skin of face, hands, legs, feet etc. First, upper dermis gets infected followed by subcutaneous lymphatic vessels resulting in rashes, inflammation, leathery skin)

There are reports that smoking and alcohol increase the chances of gangrene since these may lead to arteriosclerosis resulting restriction in blood flow through arteries.

Many times, gangrene in fingers results in brain fever (meningitis), malaria, typhoid fever, diphtheria, pneumonia etc.

\section{Types of gangrene}

Mainly following three different types of gangrene have been reported:
1. Dry gangrene
2. Wet gangrene
3. Gas gangrene

\section{Dry Gangrene}

Here, blood supply in a specific part of the body becomes slow resulting in ischemic tissue and subsequently may convert into coagulative necrosis (accidental cell death).
Under the condition, no change in supply of blood from veins to heart takes place. It is often due to peripheral artery disease or acute limb ischemia. Persons with hypercholesterolemia, diabetes or arteriosclerosis have more chance of dry gangrene. Due to slow supply of oxygen in the ischemic part, process of rotting becomes slow and bacteria is unable to survive resulting in dryness in the part with shrunken and reddish-black appearance. If affected (gangrenous) part is not removed by surgery, it may fall off.

Symptoms: In dry gangrene, patient gets much severe pain in the affected part although it does not have infection. Sometimes, patient feels burning in the affected part. In most of the cases, dry gangrene is not a threat to life. If proper care and medication is continued, patient may get relief.

\section{Wet Gangrene}

Wet gangrene develops due to saprogenic bacterial infection and common infecting bacteria are Clostridium perfringens, Staphylococcal sp., Klebsiella, Streptococcus sp. and Bacillus fusiformis. In this condition, there is lesser supply of oxygen and blood due to blockage of blood vessels. The affected part accumulates blood which stimulates bacterial growth. Due to bacterial infection, there is swelling in the affected part and also a bad smell. The infecting bacteria secrete toxic substances which are responsible for septic condition. Sometimes, there is removal of skin and cells start breaking. As a result, liquids from the cells spread which cause wetness in the surrounding area.

Symptoms and treatment for wet gangrene: When Streptococcus sp. infection is there, main symptoms are swelling and severe pain in the affected part, and fever. There is formation of small yellow colored vesicles on the affected part within 36 to 72 hours of infection which turn blue or black afterwards. At a later stage, these vesicles rupture resulting release of pungent smelled liquid. It occurs generally within 7 to 10 days. Mostly, antibiotics are prescribed to kill the bacterial infection. Spray of hydrogen peroxide which is a bactericide and also help in providing oxygen, is also recommended on the affected part. The spray of hydrogen peroxide is done in a specified chamber and is done by the specialist since excess amount of it is detrimental to the body. If proper treatment is not given in time, chances of death due to spread of toxic substances are there. Sometimes, an emergency salvage amputation like guillotine amputation is recommended to restrain infection to spread to other parts of the body.

\section{Gas Gangrene}

Gas gangrene also occurs due to infection of certain bacteria. It is mainly caused by the Clostridium sp. This disease is also called Clostridial Myonecrosis. Although, there 


\section{Clinical Dermatology Open Access Journal}

are many species of Clostridium which infect human beings, the main species responsible for gas gangrene is Clostridium perfringens. The other anaerobic bacteria which may cause gas gangrene are Bacteroides and Streptococci. These bacteria are mostly found in soil and faeces from where these infect human beings. If there is any wound with damaged muscles on the body, then these bacteria infect the wound and multiply rapidly. The blood vessels within the wound may get thrombosis and there is production of hydrogen and carbon dioxide in that part which accumulate there and develop gas gangrene [1,2]. Chi, et al. [3] reported that gas produced within the wound has nearly $5.9 \%$ hydrogen, $3.4 \%$ carbon dioxide, $74.5 \%$ nitrogen and $16.1 \%$ oxygen. It spreads in the affected part very rapidly.

Symptoms: Its symptoms are generally visible within 12 to 48 hours of infection. It has been reported that sometimes when patient walks, there is a sound like cracking of bone or breakage of dry wooden log from the affected part. The common symptoms in this disease are severe pain in the affected part, fever, low pulse rate, burning and reddish or brownish color of the skin. There become cracks on the skin within 36 hours of infection. As a result, dark reddish colored tissue beneath the skin becomes visible and there is release of a liquid from the tissue. There is short supply of oxygen due to thrombosis resulting in growth of Clostridium. As severity of the disease increases, color of the patient becomes pale yellow. If affected part is leg, then patient has difficulty in walk. Besides, generally, patient shows low blood pressure.

Clinical test for gas gangrene: It is recommended to collect inoculum from the suspected body part and synthetic growth medium for Clostridium is inoculated and allowed to grow under sterile conditions in order to check the presence of Clostridium. It is a Gram positive bacteria. On X-ray of the affected part, small spots as they are gas bubbles within the tissue are visible, if positive for gas gangrene. On pathological analysis of blood, there is low count of white blood corpuscles (WBCs), a condition called as leukocytosis. Besides, there is haemolysis due to rupture of RBCs, resulting in low count of RBCs. It has been speculated that upon multiplication, Clostridium or other infecting anaerobic bacteria, secretes exo-toxins, and these exo-toxins break RBCs and other cells causing necrosis and sepsis. Besides, these bacteria also secrete certain enzymes like collagenase, proteinases, DNase, hyaluronidase which cause damage to tissue. There are number of different toxins known to be secreted by various species of Clostridium.

It has been reported that various species of Clostridium secrete alpha toxin which acts as an enzyme, phospholipase C. This enzyme is detrimental for the infected human since it helps in the necrosis of the tissue by way of splitting phospholipids present within the cell membrane of the infected human. The alpha toxin is also responsible for killing WBCs. This toxin also causes bundling of platelets and also thrombosis in blood vessels.

Clostridium perfringens has been reported to secrete another toxin called as theta toxin. This toxin is a thiol activated cytolysin. It carries haemolysis in the infected part of the body which may be detected by the clear zone of haemolysis around colonies on blood agar plates. Theta toxin remains active only in the absence of oxygen. In the presence of oxygen, it becomes inactive. It also becomes inactive in the presence of cholesterol. This toxin is also able to suppress myocardial contractility and also induces shock. When in low concentration, theta toxin also induces priming and degranulation of polymorphonuclear leukocytes and functional up-regulation of polymorphonuclear leukocytes dependent adherence molecules like integrin CD11/CD18. This, in association of streptolysin- 0 and tetanolysin causes immunological reaction. This also exposes the phospholipids present in the outer cell membrane of RBCs so that alpha toxin can split these phospholipids. Therefore, alpha and theta toxins in collaboration cause haemolysis by way of breaking RBCs $[4,5]$.

There is also a specific type of gas gangrene called as Fournier gangrene where external genitalia or perineum is the affected part. This has been more reported in older males compared to women and children. It has also been found that persons suffering from diabetes and alcoholic are more prone to Fournier gangrene. It has been speculated that male humans are 40 times more prone to this disease compared to females. This disease was first reported in 1764 by Baurienne and has been named after the name of French Venereologist, Jean Alfred Fournier who reported five cases in his lectures in 1883. The initial symptoms for this disease are swelling and severe pain in scrotum, fever, pallor and weakness. This disease occurs due to infection of bacteria like Clostridium perfringens, Staphylococcus aureus, Vibrio vulnificus and Streptococcus sp. It is diagnosed by X-ray, ultrasound or CT scan when gas bubble are visible below the skin. Intravenous antibiotics are mostly prescribed to treat the condition. When in advanced stage, surgical removal is also done. Mortality rate in this disease is nearly 20 to $40 \%$ (https://en.wikipedia.org/wiki/Fournier_gangrene).

Kiu and Hall [6] highlighted that Clostridium perfringens secretes more than 20 different toxins. They also showed that this bacterium is also responsible for intestinal diseases not only in human but also in other animals. They discussed phenotypic as well as genomic characteristics of this bacteria and its involvement in many diseases. It has also been mentioned that it got resistance against antibiotics.

Shaw, et al. [7] studied colonization of Clostridium 


\section{Clinical Dermatology Open Access Journal}

perfringens in a cohort of prematurely born neonatal infants. They showed that this bacterium is part of gut microbiome and is responsible for life threatening necrotising enterocolitis in premature infants. They also pointed out about diversity of strains in this bacterium. They selected 333 infants for study and found that $29.4 \%$ infants were colonized with this bacterium before leaving the hospital. They showed that three factors namely increased duration of maternal milk feeds, antibiotic treatment and continuous positive airway pressure (CPAP) oxygen treatment were inversely associated with probability of carriage. It was also shown that infected infants had toxin genes for collagenase, beta, beta 2, bec A/B, net $B$, perfringolysin $O$ and enterotoxin genes. However, no infant was found having necrotising enterocolitis.

Zaragoza, et al. [8] discussed the status of toxins, diseases and toxoid production in various pathogenic Clostridium species including Clostridium perfringens. They showed that many pathogenic strains of Clostridium produce exo-toxins which are responsible for many fatal diseases resulting deaths of humans and are also responsible for revenue loss in agricultural sector. They described diseases like tetanus, gas gangrene, enterotoxemia, nectrotic enteritis and pseudomembranous colitis which are caused by various pathogenic strains of Clostridium.

Treatments: For gas gangrene, generally intravenous antibiotics injections are prescribed. If symptoms of cellulitis are visible, then surgeon has to remove the affected part by surgery and after surgery, hydrogen peroxide spray is used to avoid spreading of infecting bacteria in other parts of the body. Gas gangrene is more dangerous compared to dry and wet gangrenes. If proper care is not done, sometimes due to stoppage of blood supply and comma, there may be death of the patient.

\section{Conclusion}

Gangrene is a dreaded disease where necrosis of the affected part takes place. There are mainly three different types of gangrene namely dry, wet and gas gangrene. Out of these, dry gangrene is not caused due to bacterial infection whereas, wet and gas gangrenes are caused by bacterial infections. Gas gangrene is most dreaded disease where many deaths take place or surgical removal of the affected part is more common. Fournier gangrene is a specific gas gangrene where external genitalia or perineum are affected.
People must take precautions to avoid infection of pathogenic bacteria like Clostrium perfringens, Streptococcus sp. etc.

\section{Acknowledgements}

Author acknowledges the facilities of the Department of Biotechnology, Ministry of Science and Technology, Government of India, New Delhi (DBT) present in the Department under the Bioinformatics Sub Centre as well as M.Sc. Biotechnology program, and used in the present work.

\section{References}

1. Yang Z, Hu J, Qu Y, Sun F, Leng X, Li H, Zhan S (2015) Interventions for treating gas gangrene. Cochrane Database Syst Rev 12: CD010577.

2. Sakurai J, Nagahama M, Oda M (2004) Clostridium perfringens alpha-toxin: characterization and mode of action. Journal of Biochemistry 136(5): 569-574.

3. Chi CH, Chen KW, Huang JJ, Chuang YC, Wu MH (1995) Gas composition in Clostridium septicum gas gangrene. Journal of the Formosan Medical Association 94(12): 757-759.

4. Stevens DL, Bryant AE (1993) Role of theta toxin, a sulfhydryl-activated cytolysin, in the pathogenesis of clostridial gas gangrene. Clinical and Infectious Diseases 16(4): 195-199.

5. Stevens DL, Mitten J, Henry C (1987) Effects of alpha and theta toxins from Clostridium perfringens on human polymorphonuclear leukocytes. Journal of Infectious Diseases 156(2): 324-333.

6. Kiu R, Hall LJ (2018) An update on the human and animal enteric pathogen Clostridium perfringens. Emerging Microbes and Infections 7(1): 141.

7. Shaw AG, Cornwell E, Sim K, Thrower H, Scott, H, et al. (2020) Dynamics of toxigenic Clostridium perfringens colonisation in a cohort of prematurely born neonatal infants. BMC Pediatrics 20: 75.

8. Zaragoza NE, Orellana CA, Moonen GA, Moutafis G, Marcellin E (2019) Vaccine production to protectanimals against pathogenic Clostridia. Toxins 11(9): 525. 